\title{
Isolation, characterization and antibiotic susceptibility of staphylococcal isolates, with special reference to methicillin resistant Staphylococcus aureus, from anterior nares of health care workers in a tertiary health care centre.
}

\section{Mukul Chaurasia}

JLN Medical College, Ajmer, Rajasthan, India

\section{Neha Agrawal}

JLN Medical College, Ajmer, Rajasthan, India

Ankita Chourasia

Maharshi Dayanand Saraswati University, Ajmer, Rajasthan, India

Monica Bhatnagar

Maharshi Dayanand Saraswati University, Ajmer, Rajasthan, India

Geeta Parihar

JLN Medical College, Ajmer, Rajasthan, India

Vijaylatha Rastogi

JLN Medical College, Ajmer, Rajasthan, India

Amit Tak ( $\sim$ dramittak@gmail.com )

ICMR- National Centre for Disease Informatics and Research, Bengaluru, Karnataka, India

\section{Research Article}

Keywords: antibiotic susceptibility pattern, health care workers, methicillin resistant Staphylococcus aureus, minimum inhibitory concentration, nosocomial infections.

Posted Date: March 8th, 2021

DOI: https://doi.org/10.21203/rs.3.rs-304840/v1

License: (c) (1) This work is licensed under a Creative Commons Attribution 4.0 International License. Read Full License 


\section{Abstract}

Background: Staphylococcus aureus (S.aureus) and its resistant form Methicillin resistant S. aureus (MRSA) is one of the most common nosocomial pathogen, causing a wide range of infections in humans. The anterior nares are the main ecological niche for S.aureus. Nasal carriage of $S$. aureus acts as an important reservoir of infection among the colonized health care workers and they transmit the infection to the community. The aim of the present study is to estimate nasal colonization of S.aureus (with special reference to MRSA) in health care workers (doctors and nursing staff) and its antibiotic susceptibility pattern.

Methods: A descriptive study was planned in the Department of Microbiology, JLN Medical College, Ajmer (Rajasthan, India) after due approval from institutional ethics committee. A total of 170 health care workers of either sex and ages between 18 to 60 years were screened for S.aureus. Identification was done using standard microbiological techniques, by studying their morphology, colony and biochemical characteristics. MRSA was detected by cefoxitin disc diffusion test, oxacillin disc diffusion test, minimum inhibitory concentration (MIC) of oxacillin by E-test and oxacillin screen agar test. The observations were described in proportions and chi-squared test was used to find independence. Statistical significance was considered at $5 \%$.

Results: Among 170 samples, 159 (93.53\%) samples (50 doctors and 109 nursing staff) had staphylococci colonization. Out of 159 isolates, 34(21.38\%) isolates were S.aureus. Further, 8(5.03\%) S. aureus isolates were resistant to both cefoxitin and oxacillin and had oxacillin MIC values $\geq 4 \mu \mathrm{g} / \mathrm{ml}$ and were considered MRSA. All the MRSA belonged to nursing staff [Males: $5.50 \%$, Females : $1.83 \%$ ]. All $S$. aureus and MRSA isolates were found sensitive to linezolid. In addition, $0.63 \%$ MRSA was found vancomycin intermediate $S$. aureus (VISA) and all MRSA isolates were sensitive to mupirocin (minimum inhibitory concentration $\leq 4 \mu \mathrm{g} / \mathrm{ml}$ ).

Conclusion: Screening and treatment of health care workers colonized with MRSA should be an important component of hospital infection control policy. These measures will prevent spread of infection to patients and community and thereby reduce the morbidity, mortality and health care costs associated with nosocomial infections.

\section{Introduction}

Staphylococci are ubiquitous colonizers of skin and mucosa and highly successful opportunistic pathogens. S. aureus is one of the most harmful species of staphylococci encountered ${ }^{[1]}$. It is one of the most pathogenic bacterial species in humans causing a wide variety of infections ranging from mild skin and soft tissue infections (furuncles, carbuncles etc) to severe life-threatening infections like chronic bone infections, necrotizing pneumonia, bacteremia, septicemia, acute endocarditis, myocarditis, pericarditis, osteomyelitis, encephalitis, meningitis, chorioamnionitis, mastitis, toxic-shock syndrome, scalded skin syndrome ${ }^{[2-5]}$ and intravenous or other sites where tubes enter the body (indwelling medical 
devices) ${ }^{[6]}$. It is distinct from coagulase-negative staphylococci (CoNS) e.g. S. epidermidis, and is more virulent despite phylogenic similarities between them ${ }^{[7,8]}$.

The key characters of $S$. aureus are colony pigmentation, production of free coagulase, clumping factor, protein-A, heat-stable nuclease, lipase and acid production from mannitol ${ }^{[3]}$. The species aureus, refers to the fact that colonies often have a golden color when grown on solid media, while CoNS form pale, translucent, white colonies.

Staphylococcal infections occur frequently in hospitalized patients and have severe consequences, despite antibiotic therapy ${ }^{[9]}$. $S$. aureus are generally susceptible to $\beta$-lactam antibiotics, but extensive use of this class of drugs has led to increasing emergence of resistant strains ${ }^{[10]}$. The most notable example is the emergence of Methicillin-resistant Staphylococcus aureus (MRSA), which was reported just one year after the introduction of methicillin ${ }^{[11]}$. Also known as "a superbug" MRSA has become a major problem in most medical institutions because it is creating life-threatening situations ${ }^{[11]}$. MRSA is a major healthcare-associated (HA-MRSA) as well as a community-associated (CA-MRSA) infection [6].

Healthcare Workers (HCWs) constitute an important reservoir of $S$. aureus. Nasal carriage of $S$. aureus acts as an important reservoir of infection among those colonized, who may then transmit the infection to co-workers and others in the community ${ }^{[12]}$. Approximately $20 \%$ of individuals are persistent carriers, about $60 \%$ are intermittent carriers and $20 \%$ almost never carry $S$. aureus ${ }^{[13]}$. Several studies have reported that the rate of the nasal carriage of $S$. aureus among the HCWs ranges from $16.8 \%-56.1 \%$ [14-17]. Studies conducted in different hospital settings worldwide including India, have reported the prevalence of MRSA in HCWs in the range of $5.8 \%$ to $17.8 \%[18-22,9,12]$. The growing problem in India is that MRSA prevalence has increased from $12 \%$ to $80.83 \%{ }^{[23]}$. The health care workers who are found to be colonized with S.aureus are advised to apply mupirocin ointment in their anterior nares and they should be retested for the nasal carriage of S. aureus after 3 months of treatment ${ }^{[9]}$.

The aim of the present study is to estimate the nasal carriage and antimicrobial susceptibility pattern of Staphylococcus aureus and MRSA isolates among the HCWs in a tertiary health care centre. The prevalence of S.aureus carriers and its resistance to methicillin will help the institution develop a better MRSA infection control policy.

\section{Materials And Methods}

This descriptive study was carried out in the Department of Microbiology, Jawahar Lal Nehru Medical College and Hospital, Ajmer from November 2016 to December 2017. The study was approved from the Ethics Committee of J.L.N. Medical College, Ajmer and written informed consent was obtained from all the participants.

A total of 170 health care workers (HCWs) aged 18 to 60 years, actively involved in health care provision in different departments of J.L.N. Medical College were enrolled for the study. Each participant was 
interviewed using a questionnaire concerning general socio-demographic information, personal details and clinical symptoms. Exclusion criteria included health care workers not actively involved in patient care or suffering from underlying chronic disease or respiratory tract infections, history of recent hospitalization, intake of broad spectrum antibiotics, fever or those who refused consent.

\section{Sample collection}

Nasal swabs from anterior nares of both nostrils were collected using sterile cotton swabs with transport tube. A swab pre-moistened with sterile saline was inserted approximately 1-2 cm into the anterior nares and slowly rotated against the nasal mucosa five times ${ }^{[24]}$. Both nostrils were sampled using the same swab. After collection swabs were re-inserted in the transport tube, labeled properly and transported to the laboratory within 30 minutes of collection for further processing.

\section{Sample processing}

All the specimens were inoculated on 5\% sheep blood agar, nutrient agar and MacConkey agar (HiMedia Laboratories Pvt. Ltd. Mumbai, Maharashtra, India) and incubated at $37^{\circ} \mathrm{C}$ for 24 hours. After incubation, identification of genus Staphylococcus was done using standard microbiological techniques, by studying their morphology, colony characteristics and biochemical properties. Staphylococci were identified as Gram positive, catalase positive, furazolidone susceptible and bacitracin resistant. $S$. aureus colonies were further identified as slide and tube coagulase positive, polymyxin B resistant and mannitol fermenting giving yellow pigmentation on mannitol salt agar.

\section{Antimicrobial susceptibility testing}

Antibiotic susceptibility was studied by modified Kirby-Bauer disc diffusion method ${ }^{[25]}$ on Mueller Hinton Agar plates (120 mm diameter) using commercially available antibiotic discs (HiMedia Laboratories Pvt. Ltd. Mumbai, Maharashtra, India): penicillin G (10 units), cephalexin (30 $\mu \mathrm{g})$, cefoxitin $(30 \mu \mathrm{g})$, oxacillin $(1 \mu \mathrm{g})$, gentamicin $(10 \mu \mathrm{g})$, netilmicin $(30 \mu \mathrm{g})$, ciprofloxacin $(5 \mu \mathrm{g})$, ofloxacin $(5 \mu \mathrm{g})$, levofloxacin $(5 \mu \mathrm{g})$, erythromycin $(15 \mu \mathrm{g})$, clindamycin $(10 \mu \mathrm{g})$, tetracycline $(30 \mu \mathrm{g})$, co-trimoxazole $(25$ $\mu \mathrm{g})$, quinupristin dalfopristin $(15 \mu \mathrm{g})$, vancomycin $(30 \mu \mathrm{g})$, linezolid $(30 \mu \mathrm{g})$, cephalothin $(30 \mu \mathrm{g})$, amoxyclave $(30 \mu \mathrm{g})$ and ampicillin $(10 \mu \mathrm{g})$. Zone diameter interpretation for determining sensitive, intermediate or resistant isolates was done as per CLSI 2016 guidelines ${ }^{\text {[26]. }}$

\section{Detection of methicillin-resistant Staphylococcus aureus (MRSA)}

All confirmed $S$. aureus isolates were tested for detection of methicillin resistance by four different methods. Kirby-Bauer disc diffusion method using oxacillin $1 \mu \mathrm{g}$ and cefoxitin $30 \mu \mathrm{g}$ discs (HiMedia Laboratories, Mumbai, Maharashtra, India) [27] , minimum inhibitory concentration (MIC) testing of oxacillin by E-test and growth on Oxacillin Resistance Screening Agar (ORSA) plates ${ }^{[28]}$ as per CLSI 2016 guidelines ${ }^{[26]}$. Zone of inhibition of size of $\leq 10 \mathrm{~mm}$ was taken as resistant, 11-12 as intermediate and $\geq 13 \mathrm{~mm}$ as sensitive for oxacillin. Zone of inhibition of size of $\leq 21 \mathrm{~mm}$ was taken as resistant, and $\geq 22$ 
$\mathrm{mm}$ as sensitive for cefoxitin. On oxacillin E-test, an MIC of $\leq 2 \mu \mathrm{g} / \mathrm{ml}$ was considered susceptible and $\geq$ $4 \mu \mathrm{g} / \mathrm{ml}$ as resistant. Any growth on oxacillin screen agar was considered as methicillin (oxacillin) resistant.

\section{Detection of mupirocin resistant Staphylococcus aureus}

The MIC of mupirocin for isolation of S.aureus (Mupirocin resistance) was determined by Epsilometer test (E-test) using HiMedia, mupirocin strip (range 0.064-1024 $\mu \mathrm{g} / \mathrm{ml}$ ) and interpreted as per CLSI 2016 guidelines $^{[26]}$. Isolates with mupirocin MICs $\geq 512 \mu \mathrm{g} / \mathrm{ml}$ were considered as high-level resistant (MuH), those with MICs 8-256 $\mu \mathrm{g} / \mathrm{ml}$ were considered as low-level resistant (MuL), and with $\leq 4 \mu \mathrm{g} / \mathrm{ml}$ were considered as mupirocin sensitive.

Statistical analysis: The descriptive statistics for quantitative data was expressed as mean and standard deviation and qualitative data was expressed as proportions. Chi-squared test was used to find independence of attributes at $5 \%$ level of significance (. The JASP 0.11.1.0 statistical package was used for statistical analysis.

\section{Results}

In the present study, nasal swabs were randomly collected from a total of 170 health care workers (HCWs) from various clinical departments and screened for the study of Staphylococcus colonization. Among 170 samples, 159 (93.53\%) had staphylococci colonization. Out of these 159 HCWs, with age ranging between 18 to 60 years, $99(62.26 \%)$ were males and $60(37.74 \%)$ were females. The colonization rate was $31.45 \%, 34.59 \%, 18.24 \%$ and $15.72 \%$ in the age groups ' $18-30$ ', ' $31-40$ ', '41-50' and ' $51-60$ ' years respectively (Figure 1 ).

Out of 159 subjects, 50 were doctors and 109 were nursing staff. Out of 50 doctors, 37 (74\%) were males and 13 (26\%) were females. Among 109 staff, 62 (56.88\%) were males and 47 (43.12\%) were females. In doctors, the maximum carriage rate was observed in the age group $31-40$ years i.e. $60 \%$, where $50 \%$ were males and 10\% were females. In nursing staff group, maximum carriage was seen in 18-30 years age group where $20.18 \%$ were males and $18.35 \%$ were females accounting for a total of $38.53 \%$ carriage rate in their group (Figure 1).

In the present study Staphylococcus colonization was detected in 159 (93.53\%) health care workers which comprised of $34(21.38 \%)$ S. aureus and 125 (78.61\%) CoNS isolates. Dual colonization with S. aureus and CoNS was observed in 10 samples. The carriage rate of $S$. aureus was significantly higher in nursing staff $(26.60 \%)$ as compared to doctors $(10 \%) \quad\left[c^{2}=5.62, p=0.018\right]$. Professors/associate professors/assistant professors and resident doctors were found to have $S$. aureus nasal carriage rate $16.67 \%$ and $7.89 \%$ respectively (Figure 1 ).

Antimicrobial sensitivity pattern of Staphylococcus isolates by disc diffusion method is shown in Figure 2. Ten subjects had concomitant colonization of $S$. aureus and CoNS. Therefore antimicrobial 
susceptibility testing was done for 169 Staphylococcus isolates.

Among the antibiotics tested, all the staphylococcal isolates were susceptible only to linezolid (100\%). Maximum resistance was shown to penicillin G (97.49\%). Resistance for cefoxitin and oxacillin was 4.73 $\%$ and $5.92 \%$ respectively.

All the $S$. aureus isolates were found to be susceptible to linezolid (100\%). All S. aureus isolates showed complete resistance to penicillin G $(100 \%)$. Extremely low susceptibility was shown for erythromycin $(17.71 \%)$ and cotrimoxazole (17.65 \%). Resistance to cefoxitin and oxacillin was $23.53 \%$ (Figure 3 ).

Detection of MRSA was done by four different phenotypic methods. Among $34 \mathrm{~S}$. aureus isolates studied, 8 isolates $(23.53 \%)$ were found to be MRSA. While in oxacillin screen agar testing, $6(17.65 \%)$ isolates were found to be MRSA. No isolate showed intermediate resistance. Thus out of $34 \mathrm{~S}$. aureus isolates, 8 $(23.53 \%)$ were MRSA and 26 (76.47\%) were methicillin sensitive S. aureus (MSSA) (Table 1). One MRSA isolate showed intermediate resistance to vancomycin and was labeled as vancomycin intermediate Staphylococcus aureus (VISA).

In the present study, the $8 \mathrm{~S}$. aureus isolates that were resistant to both cefoxitin and oxacillin had oxacillin MIC values $\geq 4 \mu \mathrm{g} / \mathrm{ml}$ (Table 2). There were no isolates found resistant to cefoxitin and intermediate resistance to oxacillin at the same time. No isolate was found resistant to cefoxitin while being sensitive to oxacillin. $26 \mathrm{~S}$. aureus isolates that were sensitive to both oxacillin and cefoxitin by disc diffusion method had MICs $\leq 2 \mu \mathrm{g} / \mathrm{ml}$, indicating their susceptibility to oxacillin. Thus out of $34 \mathrm{~S}$. aureus isolates, 8 (23.53\%) were MRSA and the remaining 26 (76.47\%) were oxacillin and methicillin susceptible (MSSA).

Out of 159 isolates, $21.38 \%$ subjects had S. aureus colonization out of which $5.03 \%$ had MRSA colonization (Table 3 ). All of these MRSA carriers belonged to nursing staff. The carriage rate of $S$. aureus too was significantly more in nursing staff i.e. $26.60 \%$ with MRSA carriage rate of $7.34 \%$.

Professors/associate professors/assistant professors and resident doctors were found to have $S$. aureus nasal carriage rate $16.67 \%$ and $7.89 \%$ respectively with no MRSA carriage. All MRSA carriers belonged to nursing staff. The MRSA carriage rate was $5.5 \%$ and $1.83 \%$ in male and female nursing staff respectively (Table 4).

For $S$. aureus, mupirocin MIC $\leq 4 \mu \mathrm{g} / \mathrm{ml}$ is considered as susceptible. MIC $8-256 \mu \mathrm{g} / \mathrm{ml}$ is considered as intermediate resistant and MIC $\geq 512 \mu \mathrm{g} / \mathrm{ml}$ is considered as resistant. All $S$. aureus isolates had MIC $\leq 4$ $\mu \mathrm{g} / \mathrm{ml}$ for mupirocin, indicating mupirocin susceptibility of all the isolates (Table 5). Further, as many as 13 S. aureus and 1 MRSA isolate had MIC $\leq 0.125 \mu \mathrm{g} / \mathrm{ml}$ and 11 S. aureus and 3 MRSA isolates had MIC $\leq 0.25 \mu \mathrm{g} / \mathrm{ml}$.

\section{Discussion}


Staphylococcus aureus is a common component of the skin flora, and $30 \%$ to $50 \%$ of healthy adults are colonized with it at any given time. The primary site of colonization of $S$. aureus in humans are the anterior nares $[2,29]$. Hospital workers have higher rates of MRSA nasal colonization than the general population [30]. In the present study $21.38 \%$ subjects had S. aureus colonization. Among HCWs around the globe, the nasal carriage rates of S.aureus have been reported as $14 \%$ in Nigeria, $27.5 \%$ in Turkey, $31.1 \%$ in Iran, 33.4\% in France and 39.3\% in Spain [31]. The growing problem in India is that MRSA prevalence has increased from $12 \%$ to $80.83 \%$ [23].

In a total of 159 subjects (50 doctors and 109 nursing staff) S. aureus and CoNS appeared in 34 and 125 samples respectively. Dual colonization of $S$. aureus and CoNS was observed in 10 samples. However there was no dual isolation from any of the swab in a study conducted by Vinodhkumaradithyaa, Uma et al. $2009{ }^{[22]}$. The prevalence of the $S$. aureus nasal carriage was higher among the male HCWs (13.21\%) than the females (8.18\%). Similar observation was reported by Rongpharpi, Hazarika et al. (2013) ${ }^{[9]}$. The carriage rate of $S$. aureus was significantly more in nursing staff $26.60 \%$. Professors/associate professors/assistant professors and resident doctors were found to have $S$. aureus nasal carriage rate $16.67 \%$ and $7.89 \%$ respectively. Out of 34 S. aureus $5.03 \%$ had MRSA colonization. All of these MRSA carriers belonged to nursing staff with MRSA carriage rate of $7.34 \%$.

Similar studies from Barabanki, Uttar Pradesh reported 81\% Staphylococcus , 48\% S. aureus and 14\% MRSA colonization ${ }^{[12]}$. However, Bhatiani, Yadav et al. (2017) reported $39 \%$ and $15 \%$ carriage rates of $S$. aureus and MRSA in Rama Medical College, Hospital and Research Center, Kanpur which is almost similar to our findings ${ }^{[32]}$. Shobha, Rao et al. (2005) found none of the health care workers colonized with S. aureus [33] while a study from south India showed $9.3 \%$ S.aureus colonization ${ }^{[34]}$.

On MRSA detection using oxacillin disc diffusion, cefoxitin disc diffusion and MIC of oxacillin by E-test, 8 $(23.53 \%)$ isolates were found to be MRSA. In our study, 8 S. aureus isolates that were resistant to both cefoxitin and oxacillin had oxacillin MIC value $\geq 4 \mu \mathrm{g} / \mathrm{ml}$ and 26 isolates that were sensitive to both oxacillin and cefoxitin had MIC values $\leq 2 \mu \mathrm{g} / \mathrm{ml}$. Oxacillin screen agar could detect only $6(17.65 \%)$ isolates instead of 8 detected by other three methods. Hence it is recommended that all four methods be used for detection of oxacillin resistance. Pramodhini, Thenmozhivalli et al. (2011) found oxacillin disc diffusion method to be less sensitive for the detection of MRSA ${ }^{[35]}$. Mohanasoundaram and Lalitha (2008) obtained $100 \%$ concordance in disc diffusion method and oxacillin MIC using agar dilution methods ${ }^{[36]}$.

In the present study, $97.04 \%$ staphylococcal isolates and $100 \% \mathrm{~S}$. aureus and MRSA were found to be resistant to penicillin G. Similar findings were observed by Bala, Aggrawal et al. (2010) and Bhatiani, Yadav et al. (2017) where penicillin was found to be $100 \%$ resistant to all strains of $S$. aureus ${ }^{[37,32]}$, but Rongpharpi, Hazarika et al. (2013) reported 90\% ${ }^{[9]}$, Duran, Ozer et al. (2012) reported $92.8 \%{ }^{[38]}$, Kandle, Ghatole et al. 2003 reported $98.9 \%$ penicillin resistance ${ }^{[39]}$. All the MRSA isolates were resistant to penicillin as reported by Agarwal, Singh et al. (2015) ${ }^{[12]}$. Ampicillin and amoxyclav showed a resistance 
of $31.95 \%$ and $37.28 \%$ for staphylococci and $55.88 \%$ and $67.65 \%$ for $S$. aureus. Out of 8 MRSA isolates, $6(75 \%)$ and $8(100 \%)$ isolates were found to be resistant to ampicillin and amoxyclav respectively. Bhatiani, Yadav et al. 2017 has reported a $100 \%$ resistance to ampicillin ${ }^{[32]}$ while $88.57 \%$ and $82.0 \%$ resistance to ampicillin by $S$. aureus isolates was observed by Rongpharpi, Hazarika et al. (2013) and Jindal, Malhotra et al. (2016) in studies conducted among HCWs respectively $[9,40]$. Study conducted at a tertiary care hospital in Iran reported $89.4 \%$ resistance among MRSA ${ }^{[41]}$.

$34.91 \%$ Staphylococcus isolates, $64.71 \%$ S. aureus isolates and all MRSA isolates were resistant to cephalexin while in another study $73.7 \%$ MRSA isolates were found to be resistant to cephalexin in a similar study ${ }^{[42]}$. $24.85 \%$ and $15.38 \%$ staphylococcal, $50 \%$ and $38.24 \%$ S.aureus, $87.50 \%$ and $75.00 \%$. MRSA isolates were resistant to gentamicin and netilmicin respectively. In studies by Hauschild, Sacha et al. (2008) and Schmitz, Fluit et al. (1999) $24.4 \%$ and $23 \%$ resistance was shown in S. aureus isolates to the above aminiglycosides ${ }^{[43,44]}$. In our study of the $34 S$. aureus isolates, $38.24 \%$ were resistant to at least one of the two aminoglycosides tested. Hauschild, Sacha et al. (2008) reported that $38.1 \% S$. aureus were resistant to one of the aminoglycosides tested ${ }^{[43]}$.

42.60\% Staphylococcus isolates and $58.82 \%$ S. aureus isolates were resistant to ciprofloxacin. Lower incidence of resistance(10.4\%) was reported by Tahnkiwale, Roy et al. (2002) ${ }^{[45]}, 41 \%$ by Duran, Ozer et al. (2012) ${ }^{[38]}$ and $90 \%$ by a Mexican study on 211 isolates ${ }^{[46]}$. In Europe resistance by region showed a $5.6 \%$ resistance in northern, $6.2 \%$ in central and $23.6 \%$ in southern region ${ }^{[47]}$. Resistance to ofloxacin was shown by $18.34 \%$ Staphylococcus isolates and $32.35 \%$ S. aureus isolates. Levofloxacin resistance stood at $9.47 \%$ and $11.76 \%$ for Staphylococcus and S. aureus isolates respectively. However, $87.50 \%, 75.00 \%$ and $12.50 \%$ MRSA isolates showed resistance to ciprofloxacin, ofloxacin and levofloxacin respectively. In contrast, Agarwal et al. reported 50\% MRSA isolates resistant to ciprofloxacin and $21.4 \%$ for levofloxacin ${ }^{[12]}$

Erythromycin resistant Staphylococcus often has cross resistance to other macrolides, lincosamide and streptogramin type $\mathrm{B}\left(\mathrm{MLS}_{\mathrm{B}}\right)^{[48]}$. In the present study erythromycin resistance was seen in $85.80 \%$ and $85.29 \%$ Staphylococcus and S. aureus respectively. However a lower resistance to erythromycin ranging between 66.66\%- 67.9 \% has been observed by Bhatiani, Yadav et al. (2017), Bala, Aggrawal et al. (2010) and Kausalya, Kashid et al. (2015) ${ }^{[32,37,49]}$. Clindamycin resistance was shown in $36.69 \%$ and $50 \%$ Staphylococcus and S. aureus respectively while in a study by Verma, Joshi et al. (2000) ${ }^{[23]}$, erythromycin and clindamycin resistance was found to be $52.8 \%$ and $48.28 \%$ respectively in $S$. aureus isolates. $25.44 \%$ Staphylococcus and $38.24 \%$ S. aureus isolates respectively were tetracycline resistant. A higher resistance was reported by Shittu and Lin (2006) and Duran, Ozer et al. (2012) who reported $55.9 \%$ and $35.6 \%$ resistance for $S$. aureus isolates respectively $[50,38]$. In our study, $75.00 \%(6 / 8)$ MRSA isolates were found to be resistant to tetracycline which is much higher as reported by Agarwal, Singh et al. (2015) ${ }^{[12]}$. 
During the 17 year period of the studies by Cuevas, Cercenado et al. (2004) there was low resistance of $S$. aureus to cotrimoxazole in all the studies $(0.5 \text { to } 2.1 \%)^{[51]}$. In our study, $82.35 \% \mathrm{~S}$. aureus isolates were resistant to co-trimoxazole while other studies conducted in India have reported a resistance of $63.84 \%$ $[23], 73.3 \%{ }^{[32]}, 46.1 \%{ }^{[22]}, 31.43 \%{ }^{[9]}$ and $57.1 \%{ }^{[40]}$.

Present study showed that $87.50 \%(7 / 8)$ MRSA isolates were resistant to cotrimoxazole which correlates with the study by Mohanasoundaram and Lalitha (2008) showing $82 \%$ co-trimoxazole resistance among MRSA ${ }^{[36]}$. Somewhat higher resistance was reported by Pulimood, Lalitha et al. (1996) $(97.1 \%)^{[52]}$ while low resistance in MRSA isolates was reported by Agarwal, Singh et al. (2015) (57\%) ${ }^{[12]}$.

In the present study, a total of 55.62\% (94/169) Staphylococcus isolates and $58.82 \%$ S. aureus isolates showed resistance to quinupristin dalfopristin. All the MRSA isolates $(8 / 8,100 \%)$ were found to be resistant to quinupristin dalfopristin, while in a study only $5.56 \%$ MRSA isolates were reported as resistant to quinupristin dalfopristin ${ }^{[53]}$.

In this study, $99.41 \%$ Staphylococcus isolates were found sensitive and only one (0.59\%) reported resistant (intermediate resistant) to vancomycin. S. aureus isolates showed $97.06 \%$ sensitivity to vancomycin and only one (2.94\%) reported resistant (VISA). However, no vancomycin resistance was observed in MRSA isolates in a study conducted at Kasturba Medical College, Hospital, Mangalore [54]. Complete sensitivity to vancomycin of $S$. aureus isolates was reported by Anupurba, Sen et al. (2003) and Datta, Gulati et al. $(2011){ }^{[55,56]}$.

In 2003, Assadullah, Kakru et al. (2003) reported staphylococcal isolates with intermediate susceptibility to vancomycin in India ${ }^{[57]}$. Tiwari and Sen (2006) reported two strains of VRSA in the northern parts of India ${ }^{[58]}$. Sharma and Vishwanath (2012) studied 156 MRSA isolates which were susceptible to vancomycin by disc diffusion method but, the MIC of 18 isolates was $\geq 4 \mu \mathrm{g} / \mathrm{ml}$ (VISA) ${ }^{[59]}$.

Our study showed $100 \%$ susceptibility to linezolid. Vancomycin and linezolid were found to be the most sensitive drugs against $S$. aureus in studies by Agarwal, Singh et al. (2015) and Bhatiani, Yadav et al. $(2017)^{[12,32]}$. Golan, Baez-giangreco et al. (2006) reported a significant trend in increased MRSA linezolid resistance from 2002 onwards ${ }^{[60]}$. Linezolid, a member of the new oxazolidone class of antibiotics is highly active in vitro against MRSA and has excellent oral bioavailability and constitutes the drug of choice against MRSA infection besides vancomycin. Our study supported this.

Resistance to mupirocin is being reported from across the globe with the prevalence of $0.5 \%$ in Nigeria to $14.6 \%$ in India ${ }^{[50,61]}$. Rapid resistance to mupirocin has been reported among some strains of $S$. aureus isolated from various hospitals. In the present study of $34 \mathrm{~S}$. aureus isolates, sensitivity to mupirocin was $88 \%$ with isolates having MIC $<0.5 \mu \mathrm{g} / \mathrm{ml}$. Mohajeri, Gholamine et al. (2012) reported $100 \%$ sensitivity to mupirocin in the nasal carriage isolates of the patients ${ }^{[62]}$. Though mupirocin resistance was not seen in the $S$. aureus isolates in the study by Mohajeri, Gholamine et al. 2012), the MIC of $9.2 \%$ of the isolates was as high as $4 \mu \mathrm{g} / \mathrm{ml}$ which was very close to a low level resistance $(8 \mu \mathrm{g} / \mathrm{ml})^{[62]}$. In the study by 
Saderi, Emadi et al. (2011), 6 strains had MIC $>4 \mu \mathrm{g} / \mathrm{ml}^{[63]}$. Nagarajan et al., observed all MRSA showed high level mupirocin resistance and inducible clindamycin resistance ${ }^{[64]}$. Agarwal, Singh et al (2015) reported that $4(2 \%)$ isolates were found to be mupirocin resistant of which three isolates were high levels resistant ${ }^{[12]}$. In the presence of mupirocin resistant strains, treatment with mupirocin may be ineffective, especially with high-level resistance strains. Although low-level mupirocin resistant strains can be controlled by normal dosage schedule of mupirocin but few studies suggest that treatment failure may occur. This emphasizes the importance of identification of both high and low-level resistant strains ${ }^{[65,66}$ ,67]

Simple preventive measures like hand washing, using sterile mask, gown and avoiding touching one's nose during work, should be reinforced in all health care settings. This study reiterates the need for periodic surveillance, early and accurate detection and treatment of MRSA carriers. This should be accompanied with appropriate hospital infection control measures, to prevent the nasal carriage of MRSA in hospital health care workers.

\section{Conclusion}

In the present study very high carriage rate was detected in the anterior nares which is also the commonest site for Staphylococcus colonization. The results obtained from the antibiogram of Staphylococci, S. aureus and MRSA isolates from colonized HCWs showed the increase in rates of resistance against various antibiotics. The present study demonstrates for the first time the presence of MRSA in HCWs working in this hospital and demonstrates the prevalence of the antibiotic resistance amongst them. Vancomycin resistance in Staphylococcus species is beginning to emerge as a clinical threat, yet the attention it has received serves to underscore the seriousness of the problem.

A better understanding of these issues will be a key to helping the prevention and treatment of these infections in the future and in containing the spread of these from HCWs to patients and vice versa. All the HCWs should be periodically educated and trained about the maintenance of hygiene and infection control and the effects of the use or rather, the misuse of antibiotics.

\section{Declarations}

Limitations of the study - The study enrolled health care workers from a single tertiary health care center, however, to generalize the results multi-centric studies are required.

Conflicts of interest- There are no conflicts of interest for the authors.

Funding: Not applicable

Authors' contribution: $\mathrm{MC}$ involved in planning, concept design and hypothesis generation, NA did data collection, AC did data assembly, literature review and manuscript writing, MB helped in statistical 
analysis, GP and VR helped in data interpretation and literature review, AT helped in manuscript writing and data visualization. All authors commented and finally approved the manuscript.

Ethical Statement: The authors are accountable for all aspects of the work in ensuring that questions related to the accuracy or integrity of any part of the work are appropriately investigated and resolved. The study was conducted in accordance with the Declaration of Helsinki (as revised in 2013). The study was approved by Ethical Committee of the JLN Medical College, Ajmer (No.42954-85 dated 28 -10 20-16).

\section{References}

1. Karmakar, A., Dua, et al. (2016). "Biochemical and Molecular Analysis of Staphylococcus aureus Clinical Isolates from Hospitalized Patients." Can J Infect Dis Med Microbiol 2016: 9041636.

2. Lowy, F.D. (1998) “Staphylococcus aureusinfections” N Engl J Med. 339 (8) : 520-32.

3. Murray, P. R., E. J. Baron, et al. (2003). Manual of Clinical Microbiology. Washington, DC, USA: American Society for Microbiology.

4. Centres for Disease Control and Prevention (US) (2013). Antibiotic resistance threats in the United States, 2013. Centres for Disease Control and Prevention, US Department of Health and Human Services; 2013.

5. Demir, T, N. Coplu, et al. (2016). "Comparative analysis of phenotypic and genotypic detection of methicillin resistance among Staphylococcus aureus."Indian J Pathol Microbiol 59(3): 314-7.

6. Chambers, H.F. (2003). Tracking the spread of CMRSA. APUA Newsletter 21(2): 1-5

7. Waldvogel, F.A. (1990). "Staphylococcus aureus (including toxic shock syndrome)". In: Mandell GL, Douglas RG, Bennett JE (eds.). Principles and Practice of Infectious Disease, 3rded. Churchill Livingston, London. pp 1489- 151.

8. Projan, S. J. and R. P. Novick (1997). "The molecular basis of pathogenicity". In: Crossley KB, Archer GL, eds. The Staphylococci in Human Diseases. Churchill Livingston, London. pp 55-81.

9. Rongpharpi, S. R., N, K, Hazarika, et al. (2013). "The Prevalence of Nasal Carriage of Staphylococcus AureusAmong Healthcare Workers at a Tertiary Care Hospital in Assam with Special Reference to MRSA." J Clin Diagn Res 7(2): 257-260.

10. Centers for Disease Control and Prevention. Active Bacterial Core Surveillance Report, Emerging Infections Program Network, Methicillin-Resistant Staphylococcus aureus; 2011.

11. Khan, Z., S. Faisal, et al. (2010). "The continuing threat of Methicillin Resistant Staphylococcus aureus-past, present, future," Journal of Scientific Research 40 (2): 31-34.

12. Agarwal, L., K. Singh, et al. (2015). "Nasal carriage of Methicillin- and Mupirocin-resistant $S$. aureusamong health care workers in a tertiary care hospital". J Res Pharm Pract 4(4): 182-186.

13. Kluytmans, J., A. van Belkum, et al. (1997). "Nasal carriage of Staphylococcus aureus: epidemiology, underlying mechanisms, and associated risks." Clin Microbiol Rev 10 (3):505-520. 
14. Duncan, I. B. R., A. M. Collins, et al. (1957). "Nasal carriage of Staphylococcus pyogenes by student nurses." Can. Med. Assoc J 77(11): 1001-09.

15. Paul, M. O., A. Lamikanra, et al. (1982). "Nasal carriers of coagulase positive staphylococci in a Nigerian hospital community.” Trans. R. Soc. Trop. Med. Hyg. 76(3):319-23.

16. McAnally, T. P., M. R. Lewis, et al. (1984). “Effect of rifampin and bacitracin on nasal carriers of Staphylococcus aureus." Antimicrob Agents Chemother 25(4): 22-26

17. Dan, M., Y. Moses, et al. (1992). "Carriage of Methicillin- resistant Staphylococcus aureus by nonhospitalized subjects in Israel." Infection 20: 332-35.

18. Eveillard, M, Y. Martin, et al. (2004). "Carriage of methicillin-resistant Staphylococcus aureusamong hospital employees: Prevalence, duration, and transmission to households." Infect Control Hosp Epidemiol 25: 114-20.

19. Akoua Koffi, C., K. Dje, et al. (2004). "Nasal carriage of meticillin-resistant Staphylococcus aureusamong health care personnel in Abidjan (Côte d'Ivoire) Dakar Med 49: 70-4.

20. Cesur, S. and F. Cokça. (2004). "Nasal carriage of methicillin-resistant Staphylococcus aureusamong hospital staff and outpatients." Infect Control Hosp Epidemiol 25: 169-71.

21. Mulqueen, J., F. Cafferty, et al. (2007)." Nasal carriage of meticillin-resistantStaphylococcus aureusin GPs in the West of Ireland." Br J Gen Pract 57:811-3.

22. Vinodhkumaradithyaa, A., A. Uma, et al. (2009). "Nasal carriage of methicillin-resistant Staphylococcus aureus among surgical unit staff." Jpn J Infect Dis 62(3): 228-9.

23. Verma, S., Joshi, S., Chitnis, V., Hemwani, N., Chitnis, D. 2000. Growing problem of methicillin resistant staphylococciindian scenario. Indian J. Med. Sci., 54: 535-40.

24. Baron, E. J., R. B. Thompson, et al. (2011)." Specimen collection, transport and processing: bacteriology. In Versalovic, J., Karoll, K. C., Jorgensen, J. H., Funke, G., Landry, M. L., Warnock, D. W. Eds. Maual of Clinical Microbiology. 10th Ed. Washington, DC: ASM Press p. 228-71.

25. Bauer, A. W., W. M. Kirby, et al. (1966). "Antibiotic susceptibility testing by a standardized single disk method." Am J Clin Pathol 45(4): 493-6.

26. CLSI (2016). (Clinical and Laboratory Standard Institute) “Performance Standards for Antimicrobial Susceptibility Testing." CLSI document M100S. 26th Ed. Wayne, P.A. Clinical and Laboratory Standard Institute, USA.

27. Mathews, A. A., M. Thomas, et al. (2010). "Evaluation and comparison of tests to detect methicillin resistant S. aureus." Indian J Pathol Microbiol 53(1): 79-82.

28. K. B.,P. Agrawal, et al. (2009). "Comparison of cefoxitin disc diffusion test, oxacillin screen agar, and PCR for mecA gene for detection of MRSA." Indian J Med Microbiol 27(1): 27-9.

29. van Belkum, A., D. C. Melles, et al. (2009). "Co-evolutionary aspects of human colonisation and infection by Staphylococcus aureus." Infect Genet Evol 9(1): 32-47. 
30. Godfrey, M.E. and I. M. Smith (1958). "Hospital hazards of Staphylococcal sepsis." JAMA 166: 197200.

31. Mahmoud, A. M., H. S. Albadawy, et al. (2015). "Inducible clindamycin resistance and nasal carriage rates of Staphylococcus aureus among healthcare workers and community members."Afr Health Sci 15(3): 861-67.

32. Bhatiani, A., S. Yadav, et al. (2017). "Prevalence of Carriage of Methicillin Resistance Staphylococcus aureus among Health Care Workers in a Tertiary Care Center, Kanpur (U.P), India." Int J Curr Microbiol App Sci 6(5): 739-46.

33. Shobha, K.L., P. S. Rao, et al. (2005). "Survey of Staphylococcus isolates among hospital personnel, environment and their antibiogram with special emphasis on methicillin resistance." Indian J. Med. Microbiol 23(3): 186-88.

34. Shinde, R. V., S. K. Pawar, et al. (2016). "Study of Nasal Carriage of Staphylococcus aureus with Special Reference to Methicillin Resistance among Nursing Staff." Arch Clin Microbiol 7:1.

35. Pramodhini, S., P. R. Thenmozhivalli, et al. (2011). "Comparison of Various Phenotypic Methods and mecA Based PCR for the Detection of MRSA." JCDR (Suppl-2) 5(7): 1359-62.

36. Mohanasoundaram, K.M. and M. K. Lalitha (2008). "Comparison of phenotypic versus genotypic methods in the detection of methicillin resistance in Staphylococcus"Indian J Med Res 127(1):78-84.

37. Bala, K., R. Aggrawal, et al. (2010). "Prevalence and susceptibility patteren of MRSA colonization in a teaching tertiary care centre in India." J Infect Dis Antimicrob Agents 27(1): 33-38.

38. Duran, N., B. Ozer, et al. (2012). "Antibiotic resistance genes \& susceptibility patterns in staphylococci." Indian J Med Res 135(3): 389-96.

39. Kandle, S. K.,M. P. Ghatole, et al. (2003). "Bacteriophage typing and antibiotic sensitivity pattern of Staphylococcus aureus from clinical specimen in and around Solapur (South Maharashtra)."J Commun Dis. 2003 Mar;35(1):17-23.

40. Jindal, N., R. Malhotra, et al. (2016). "Methicillin resistant Staphylococcus aureus (MRSA) in Malwa region of Punjab (North-West India)." Indian J Med Res 2143(3): 371-72.

41. Dibah, S.,M. Arzanlou, et al. (2014). "Prevalence and antimicrobial resistance pattern of methicillin resistant Staphylococcus aureus (MRSA) strains isolated from clinical specimens in Ardabil, Iran." Iran J Microbiol 6(3):163-8.

42. Japoni, A., M. Ziyaeyan, et al.(2010)."Antibacterial susceptibility patterns and cross-resistance of methicillin resistant and sensitive Staphyloccus aureus isolated from the hospitalized patients in Shiraz, Iran." Braz. J. Microbiol. vol.41 no.3 São Paulo Oct. 2010.

43. Hauschild, T., P. Sacha, et al. (2008). "Aminoglycosides resistance in clinical isolates of Staphylococcus aureus from a University Hospital in Bialystok, Poland." Folia Histochem Cytobiol 46(2): 225-8.

44. Schmitz , F. J., A. C. Fluit, et al. (1999)."The prevalence of aminoglycoside resistance and corresponding resistance genes in clinical isolates of staphylococci from 19 European hospitals."J Antimicrob Chemother 43(2): 253-9. 
45. Tahnkiwale, S. S., S. Roy, et al. (2002). "Methicillin resistance among isolates of Staphylococcus aureus : antibiotic sensitivity pattern \& phage typing." Indian J Med Sci 56(7): 330-4.

46. Calderón-Jaimes, E., L. E. Espinosa de los Monteros, et al. (2002).“Epidemiology of drug resistance: the case of Staphylococcus aureus and coagulase-negative staphylococci infections." Salud Publica Mex 44(2): 108-12.

47. Thomson, C. J. (1999). "The global epidemiology of resistance to ciprofloxacin and the changing nature of antibiotic resistance: a 10 year perspective." J Antimicrob Chemother 43 Suppl A: 31-40.

48. Peacock, S. J. (2005). "Staphylococcus". In Borriello, S. P. , Murray, P.R. , Funke, G. Eds. Topley and Wilson's Microbiology and Microbial Infections. 10th Ed. Hodder Arnold, Italy. 2: 771-832.

49. Kausalya, R. A. Kashid, et al. (2015) "Nasal carriage and antimicrobial susceptibility of Staphylococcusaureus, with special reference to methicillin resistance, in health care workers in a tertiary care hospital in south India." Sch Acad J Biosci 3(8): 720-24.

50. Shittu, A. O. and J. Lin (2006). "Antimicrobial susceptibility patterns and characterization of clinical isolates of Staphylococcus aureus in KwaZulu-Natal province, South Africa." BMC Infect Dis 6:125.

51. Cuevas, O., E. Cercenado, et al. (2004).“Evolution of the antimicrobial resistance of Staphylococcus spp. in Spain: five nationwide prevalence studies, 1986 to 2002." Antimicrob Agents Chemother 48(11): 4240-5.

52. Pulimood, T.B., M. K. Lalitha, et al. (1996). "The Spectrum of antimicrobial resistance among methicillin resistant Staphylococcus aureus (MRSA) in a tertiary care in India." Indian J Med Res 103: 212-5.

53. Kaur, D. C. and S. S. Chate. (2015) "Study of Antibiotic Resistance Pattern in Methicillin Resistant Staphylococcus aureus with Special Reference to Newer Antibiotic." J Glob Infect Dis.7(2): 78-84.

54. Radhakrishna, M., M. D'Souza, et al. (2013). "Prevalence of Methicillin Resistant Staphylococcus aureus Carriage amongst Health Care Workers of Critical Care Units in Kasturba Medical College Hospital, Mangalore, India." J Clin Diagn Res 7(12): 2697-2700.

55. Anupurba S., M. R. Sen, et al. (2003). "Prevalence of methicillin resistant Staphylococcus aureus in tertiary referral hospital in eastern Uttar Pradesh." Indian J. Med. Microbiol 21: 49-51.

56. Datta, P., N. Gulati , et al. (2011). "Evaluation of various methods for the detection of meticillinresistant Staphylococcus aureus strains and susceptibility patterns." J Med Microbiol 60 (Pt 11): 1613-6.

57. Assadullah, S., D. K. Kakru, et al. (2003).“Emergence of low level vancomycin resistance in MRSA.” Indian J Med Microbiol 21(3): 196-8.

58. Tiwari, H. K. and M. R. Sen (2006). "Emergence of vancomycin resistant Staphylococcus aureus (VRSA) from a tertiary care hospital from northern part of India." BMC Infectious Diseases 6: 156.

59. Sharma, P. and G. Vishwanath (2012). "Study of vancomycin susceptibility in methicillin-resistant Staphylococcus aureus isolated from clinical samples.” ATMPH 5 (3):178-180. 
60. Golan, Y., C. Baez-Giangreco, et al. (2006). "Trends in vancomycin susceptibility among consecutive MRSA isolates. In: Abstracts of the Fourty fourth Annual Meeting of the Infectious Diseases Society of America, Toronto, Ontario, Canada. Infectious Disease Society of America, Alexandria, VA, USA. Abstract LB-11 p.238.

61. Gadepalli, R., B. Dhawan, et al. (2007). "Mupirocin resistance in Staphylococcus aureus in an Indian hospital." Diagn Microbiol Infect Dis 58(1): 125-7.

62. Mohajeri, P., B. Gholamine, et al. (2012). "Frequency of Mupirocin Resistant Staphylococcus aureus Strains Isolated From Nasal Carriers in Hospital Patients in Kermanshah." Jundishapur J Microbiology 5(4): 560-3.

63. Saderi, H.,B. Emadi, et al. (2011). “Phenotypic and genotypic study of macrolide, lincosamide and streptogramin B (MLSB) resistance in clinical isolates of Staphylococcus aureus in Tehran, Iran." Med Sci Monit 17(2): BR48-53.

64. Abimanyu, N., S. Murugesan, et al. (2012). "Emergence of methicillin-resistant Staphylococcus aureus ST239 with high-level mupirocin and inducible clindamycin resistance in a tertiary care center in Chennai, South India."J Clin Microbio 50(10): 3412-3.

65. Hudson, I. R. (1994). "The efficacy of intranasal mupirocin in the prevention of staphylococcal infections: a review of recent experience." J Hosp Infect 27(2): 81-98.

66. Walker, E. S., J. E. Vasquez, et al. (2003). “Mupirocin-resistant, methicillin-resistant Staphylococcus aureus: does mupirocin remain effective?" Infect Control Hosp Epidemiol 24(5): 342-6.

67. Singh, A. K., V. Venkatesh, et al. (2013). "Mupirocin resistance in clinical isolates of Staphylococcus aureus in a tertiary care hospital set up in North India." Int J Med Res Health Sci 2: 840-7.

\section{Tables}

Table 1: Comparison of different phenotypic methods for detection of methicillin resistance in $S$. aureus isolates $(\mathrm{n}=34)$. 
Tests used for detection of MRSA

\begin{tabular}{|c|c|c|c|c|}
\hline S.aureus & Oxacillin (1 $\mu g)$ & Cefoxitin $(30 \mu \mathrm{g})$ & Oxacillin & Oxacillin MIC \\
\hline & disc diffusion & disc diffusion & screen agar & E-test \\
\hline Methicillin-resistant & $8(23.53)$ & $8(23.53)$ & $6(17.65)$ & $8(23.53)$ \\
\hline S.aureus (MRSA) & & & & \\
\hline Methicillin-sensitive & $26(76.47)$ & $26(76.47)$ & $28(82.35)$ & $26(76.47)$ \\
\hline
\end{tabular}

Note: percentage is shown in parenthesis

Table 2: Comparison of cefoxitin and oxacillin disc diffusion tests with

MIC of oxacillin by E-test method $(n=34)$

Results of oxacillin and cefoxitin disc diffusion $\quad$ MIC of oxacillin $\quad$ No. of isolates

$(\mu \mathrm{g} / \mathrm{ml})$

Resistant to both cefoxitin and oxacillin

$8(23.53)$

Resistant to cefoxitin and intermediate resistant to

$0(0.0)$

oxacilln

Resistance to cefoxitin and sensitive to oxacillin $\geq 4 \quad 0(0.0)$

Sensitive to cefoxitin and oxacillin

$$
<4
$$

Note: percentage is shown in parenthesis 
Table 3: Nasal carriage of $S$. aureus and MRSA among various health care workers $(n=34)$.

Health care workers

No. of nasal No. of

No. of MRSA

swabs collected S.aureus isolated isolated (\%)

(\%)

Professors, Associate Professors, 12

$02(16.67)$

$0(0.0)$

Assistant professors

Resident doctors

38

03 (7.89)

$0(0.0)$

Nursing staff

109

$29(26.60)$

$08(7.34)$

Total

159

$34(21.38)$

$08(5.03)$

Note: percentage is shown in parenthesis

Table 4: Sex wise distribution of nasal carriage of $S$. aureus and MRSA among various health care workers $(n=34)$, percentage is shown in parenthesis. 


\begin{tabular}{|c|c|c|c|c|c|}
\hline \multirow[t]{2}{*}{$\begin{array}{l}\text { Health care } \\
\text { workers }\end{array}$} & \multirow[t]{2}{*}{$\begin{array}{l}\text { No. of nasal swabs } \\
\text { collected }\end{array}$} & \multicolumn{2}{|c|}{$\begin{array}{l}\text { No of } S \text {. aureus isolated } \\
\text { (\%) }\end{array}$} & \multicolumn{2}{|c|}{$\begin{array}{l}\text { No. of MRSA } \\
\text { isolated }\end{array}$} \\
\hline & & Male & Female & Male & Female \\
\hline Doctors & 50 & $4(8)$ & $1(2)$ & 0 & 0 \\
\hline Nursing staff & 109 & $17(15.60)$ & $12(11.01)$ & $6(5.50)$ & $2(1.83)$ \\
\hline Total & 159 & $21(13.21)$ & 13 (8.18) & $6(3.77)$ & $2(1.26)$ \\
\hline & & 34 & & 8 & \\
\hline
\end{tabular}

Note: percentage is shown in parenthesis

Table 5: Mupirocin MIC of nasal isolates of $S$. aureus $(\mathrm{n}=34)$

\begin{tabular}{llll} 
S. No. & Mupirocin MIC $(\mu \mathrm{g} / \mathrm{ml})$ & No. of $S$. aureus isolates (\%) & No. of MRSA isolates (\%) \\
\hline 1 & $\mathbf{2} 0.125$ & 13 & 01 \\
2 & 0.25 & 11 & 03 \\
3 & 0.5 & 06 & 02 \\
4 & 1 & 03 & 01 \\
5 & 2 & 01 & 01 \\
6 & 4 & 00 & 00 \\
7 & 8 & 00 & 00 \\
Total & 34 & 08 \\
\hline
\end{tabular}

\section{Figures}


(a)

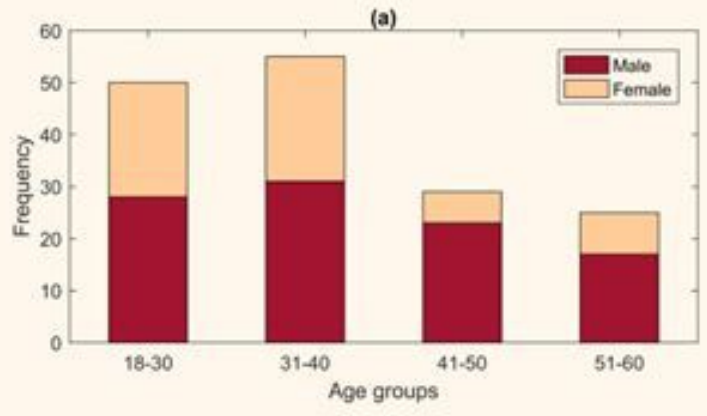

(c)

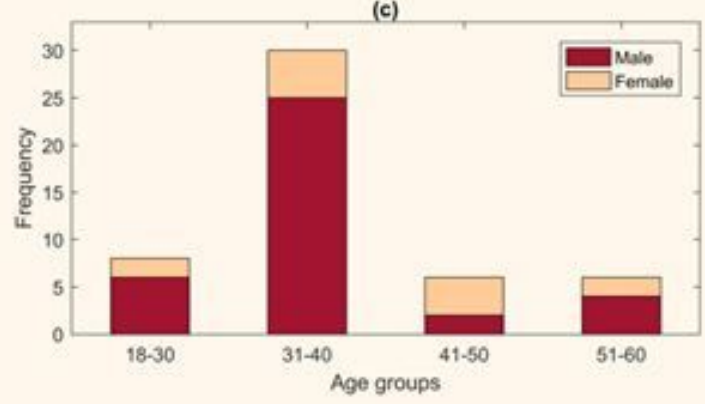

(b)

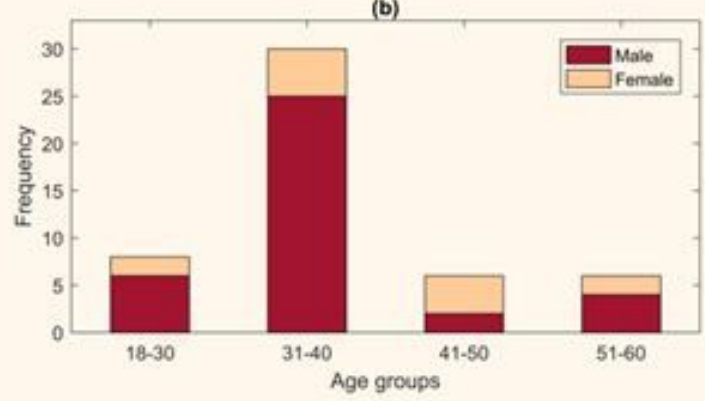

(d)

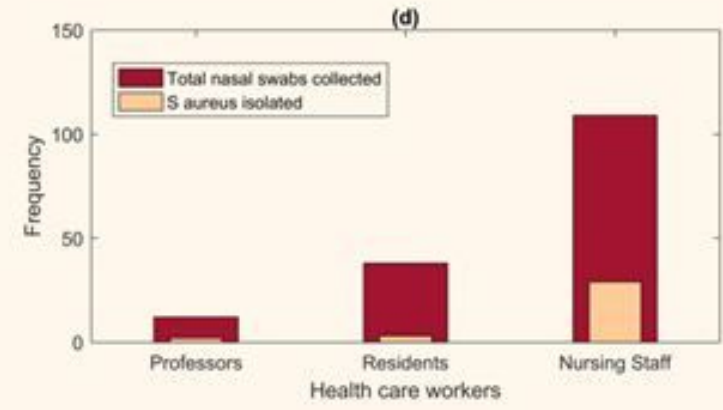

\section{Figure 1}

Stacked bar plots showing demographic profile (a) Age wise gender distribution of all health care workers $((\mathrm{N}=159)$ (b) age-wise gender distribution of doctors $(\mathrm{N}=50)$ (c) age-wise gender distribution of nursing staff $(\mathrm{N}=109)$ (d) Total number of nasal swabs collected and S.aureus isolated in health care workers $(\mathrm{N}=$ 159). 


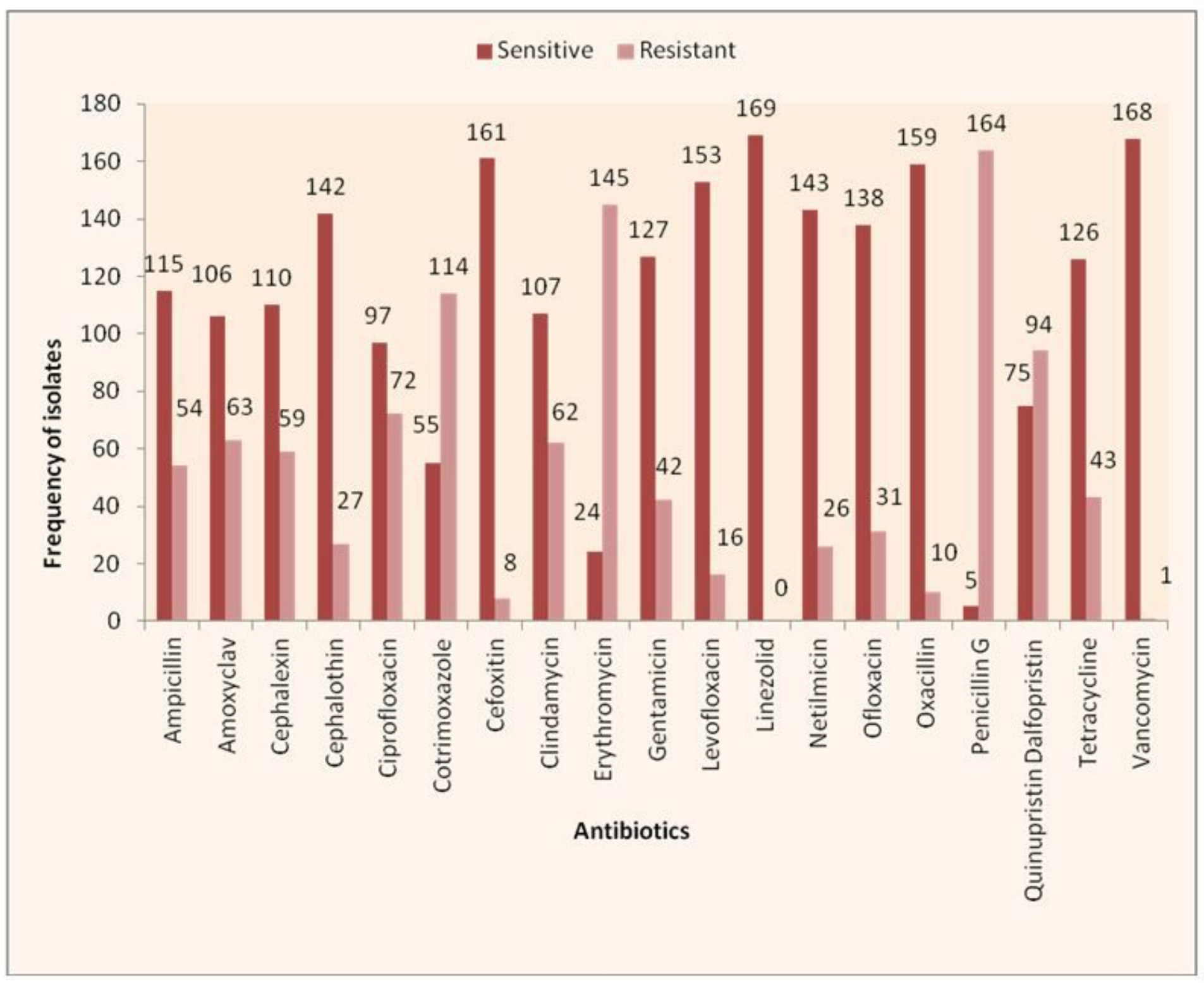

Figure 2

Bar plots showing antimicrobial sensitivity pattern of Staphylococcus isolates by modified Kirby-Bauer disc diffusion method. $(\mathrm{N}=169)$ 


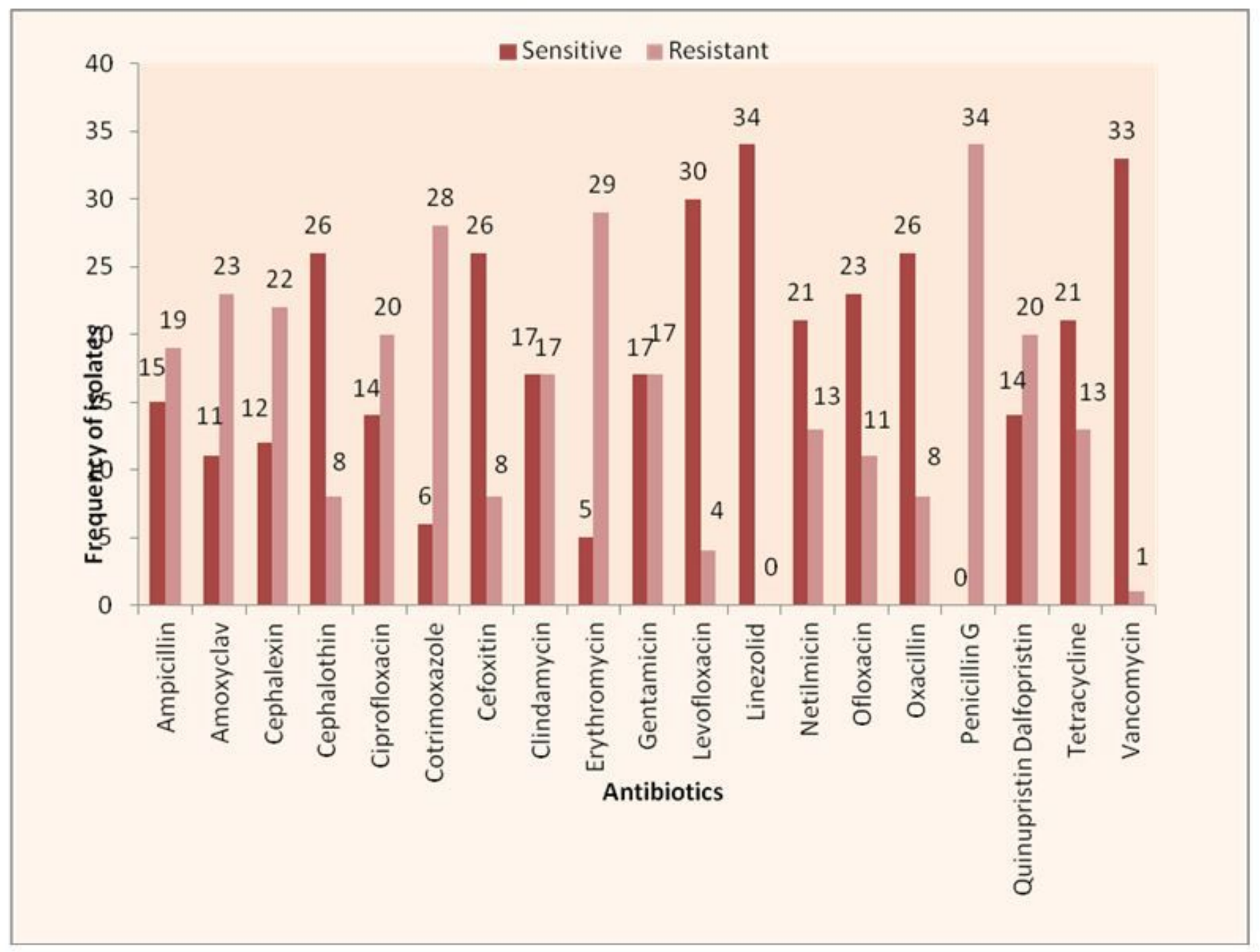

\section{Figure 3}

Bar plots showing antimicrobial sensitivity pattern of S. aureus isolates by modified Kirby-Bauer disc diffusion method. $(\mathrm{N}=34)$

\section{Supplementary Files}

This is a list of supplementary files associated with this preprint. Click to download.

- TitlePage.docx 